\title{
OPTIMIZATION STRATEGIES FOR THE VULNERABILITY ANALYSIS OF THE ELECTRIC POWER GRID *
}

\author{
ALI PINAR ${ }^{\dagger}$, JUAN MEZA ${ }^{\ddagger}$, VAIBHAV DONDE ${ }^{\S}$, AND BERNARD LESIEUTREף
}

\begin{abstract}
Identifying small groups of lines, whose removal would cause a severe blackout, is critical for the secure operation of the electric power grid. We show how power grid vulnerability analysis can be studied as a mixed integer nonlinear programming (MINLP) problem. Our analysis reveals a special structure in the formulation that can be exploited to avoid nonlinearity and approximate the original problem as a pure combinatorial problem. The key new observation behind our analysis is the correspondence between the Jacobian matrix (a representation of the feasibility boundary of the equations that describe the flow of power in the network) and the Laplacian matrix in spectral graph theory (a representation of the graph of the power grid). The reduced combinatorial problem is known as the network inhibition problem, for which we present a mixed integer linear programming formulation. Our experiments on benchmark power grids show that the reduced combinatorial model provides an accurate approximation, to enable vulnerability analyses of real-sized problems with more than 10,000 power lines.
\end{abstract}

Key words. mixed integer nonlinear programming, network inhibition, network flow, mixed integer linear programming, electric power flow, network vulnerability, graph theory

AMS subject classifications. 90C11, 90C27, 90C90, 90C30

1. Introduction. Robust operation of a power grid requires anticipation of component outages that could lead to dramatic blackouts. The current practice is to check for single contingencies to ensure the system stays intact after a single line outage. However, a small number of line outages (e.g., 3-5) can cause catastrophic blackouts, as evidenced by the Northeast Blackout in August 2003. In this article, we consider the power network vulnerability analysis problem, which aims to find small groups of lines, whose loss can cause a severe blackout. Specifically, we pose the following two related optimization problems: 1) compute the minimum number of line failures that will cause a damage of at least a specified severity and 2) compute a combination of a specified number of lines, whose loss will cause the maximum damage.

We consider the problem in a static sense by examining the relation between the operating point, which describes the current generation and consumption at each node in the network, and the feasibility boundary of the power flow equations. The severity of the events we identify could be different when dynamics and cascading events are considered. Our main focus here, therefore, is to identify simple events that can trigger a severe blackout, not to analyze its consequences, which requires solving differential algebraic equations with discrete variables. Cascading events start with a significant disturbance that forces system elements to operate beyond their capabilities. For this reason, we look for minimal changes in the network topology that push the

*This work was supported by the Director, Office of Science, Division of Mathematical, Information, and Computational Sciences of U.S. Department of Energy under contract DE-AC0376SF00098. Work also completed under Department of Energy Contract No. DE-AC02-05CH11231.

$\dagger$ Corresponding author. High Performance Computing Research Department, Lawrence Berkeley National Laboratory (apinar@lbl.gov).

${ }^{\ddagger}$ High Performance Computing Research Department, Lawrence Berkeley National Laboratory (JCMeza@lbl.gov).

$\S$ Corporate research center, ABB Inc., Raleigh NC (vaibhav.d.donde@us.abb.com). Work performed at Lawrence Berkeley National Laboratory.

๑Environmental Energy Technologies Division, Lawrence Berkeley National Laboratory and Electrical and Computer Engineering Department, University of Wisconsin, Madison. (BCLesieutre@lbl.gov) 
current operating point significantly outside of the feasibility region of the power flow equations. This problem statement leads to a bi-level optimization problem, since we are looking for minimal changes in network topology that maximize the distance between the current operating point and the new feasibility region. Moreover, the problem combines nonlinearity due to the power flow equations, with discrete variables, due to changes in the network topology.

In this article, we propose a mixed integer nonlinear programming (MINLP) formulation for the power network vulnerability analysis problem. To measure the severity of the disturbance to the system, we use a load shedding mechanism, which optimally decreases the generation and consumption in the system to restore feasibility. We avoid solving nested optimization problems by replacing the inner optimization problem that compute the distance between the current operating point and the new feasibility region, with its Karush-Kuhn-Tucker conditions. Next, we analyze the structure of a feasible solution to our MINLP formulation to reveal a special structure that can be exploited to reduce the problem to a pure combinatorial problem. We show that at a feasible solution to our MINLP formulation, the power network will be divided into two groups: one with excess generation and one with excess load, and the optimal load shedding strategy requires that in the load-rich region, we decrease only the consumption and keep the generation as is. Similarly in the generation-rich region, we decrease only the generation and keep the consumption as is. Moreover, we prove that at least one line that connects these two regions works at its maximum capacity to transfer power from the generation-rich side to the other. This clear combinatorial structure of a feasible solution means that an optimal solution seeks a decomposition with maximum load/generation mismatch and minimum transmission capability between the the two regions. This observation leads to our major result: the original MINLP problem can be reduced, after some realistic simplifications, to a pure combinatorial problem, namely the network inhibition problem. With this reduction, we directly seek the values of discrete variables in the formulation without solving the nonlinear equations, simplifying the problem complexity both in a theoretical and practical sense.

Identification of multiple contingencies has recently drawn much interest both from the optimization and power systems communities. Salmeron, Wood, and Baldick [25] employed a linearized power flow model and used a bilevel optimization framework along with mixed-integer programming to analyze the security of the electric grid. The critical elements of the grid were identified by maximizing the long-term disruption in the power system operation. The bilevel optimization framework has also been used by Arroyo and Galiana [18]. In all of these formulations the optimization framework appears promising for such types of problems where the critical system elements that make the system vulnerable to failures must be identified. Donde et al. [12], proposed a method that connected the feasibility boundary of power flow equations with spectral graph theory, when voltages are fixed at their nominal values, and only active power flow constraints are considered. Later, Donde et al. [11] extended their approach to include reactive power and proposed a mixed integer nonlinear programming formulation to identify the most significant blackout that can be caused by a specified number of lines or to identify the minimum number of lines to cause a blackout of specified severance. More recently, Lesieutre et al. [19,20] approached this problem from a graph theoretical perspective, by looking for subgraphs in a given graph that are loosely connected to the rest of the graph and have a significant load/generation mismatch. Grijalva and Sauer $[15,16]$ related topological cuts in the 
power network with the static collapse based on branch complex flows. He et. al. [17] used a voltage stability margin index to identify weak locations in a power network. Bienstock and Mattia used the direct current power flow model and mixed integer linear programming to find the most cost-effective way to increase edge capacities to avoid cascading outages for a given set of failure scenarios [3]. Oliviera et al. have used similar models and techniques to study how to add power lines to improve system resilience [21]. In addition to these largely static analyses, system dynamics for cascading events has also drawn a lot of interest. In $[4,6,8]$ Dobson et al. used a longterm model of the grid to study how failure of a component affects other components in the system, to reveal failure statistics consistent with those observed in the power grid. The same authors have also studied probabilistic models with the aim to better understand cascade propagation $[5,9,10]$.

The remainder of this article is organized as follows. Section 2 reviews matrix representations of graphs and the basics of spectral graph theory that are relevant to this article. In Section 3, we present a MINLP formulation for the power network vulnerability analysis problem. The structure of a feasible solution to this problem and how this structure can be exploited to reduce the MINLP formulation to a pure combinatorial problem are discussed in Section 4. We describe the network inhibition problem and its integer programming formulation in Section 5. Section 6 presents our experimental results, and we conclude with Section 7 .

2. Graphs and matrices. Matrix representations of graphs have long been used to apply algebraic techniques to analyze graphs. Here we review the node-arc incidence matrix and the Laplacian matrix, as two of the commonly used representations for graphs. The node-arc incidence matrix of a graph is used in flow problems, and we will use this representation to present power flow equations. The Laplacian matrix for graphs on the other hand, underlies spectral graph theory, which can be used to analyze the connectedness of graphs. Let $G=(V, E)$ be a graph with $n$ vertices and $m$ edges. We use $\left(v_{i}, v_{j}\right)$ to denote an edge that goes from vertex $v_{i}$ to vertex $v_{j}$. The node-arc incidence matrix, $A$, of this graph is an $m \times n$ matrix, where the $j$-th column of $A$ represents the $j$-th vertex, $v_{j}$, and the $i$-th row represents the the $i$-th edge, $e_{i}$, in $G$. Each row has only two nonzeros at the columns that represent the end vertices of the respective edge. The entry is -1 or 1 , depending on whether the respective edge is directed from or to the corresponding vertex, respectively. Formally, we use $a_{i j}$ to denote the matrix entry at the $i$-th row and the $j$-th column of $A$, which is defined as follows.

$$
a_{i j}= \begin{cases}-1 & \text { if } \quad e_{i}=\left(v_{j}, u\right) \in E \\ 1 & \text { if } \quad e_{i}=\left(u, v_{j}\right) \in E \\ 0 & \text { otherwise }\end{cases}
$$

The node-arc incidence matrix $A$ of the graph in Fig. 2.1 is as follows.

$$
A=\left(\begin{array}{rrrrrr}
-1 & 1 & & & & \\
-1 & & 1 & & & \\
& 1 & -1 & & & \\
-1 & & 1 & & \\
-1 & & & 1 & \\
& & -1 & & 1 & \\
& & & -1 & & 1 \\
& & & & -1 & 1
\end{array}\right)
$$




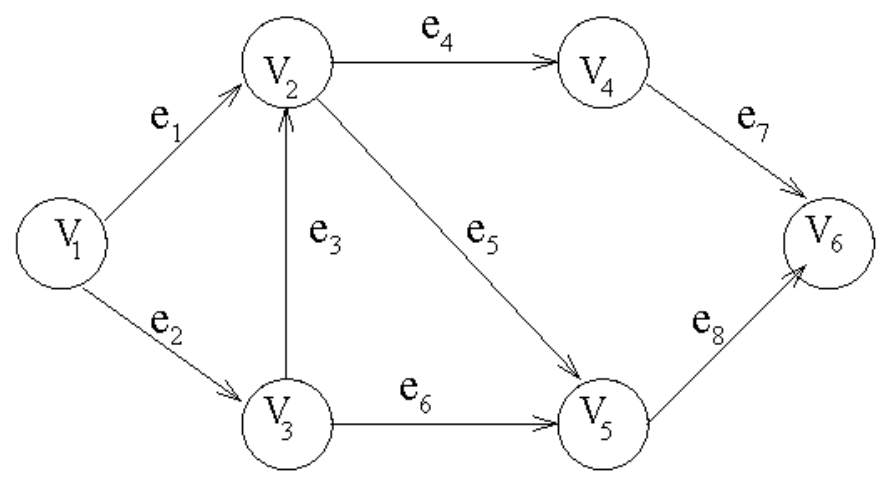

FIG. 2.1. A sample directed graph

The Laplacian of a graph $G=(V, E)$ is an $n \times n$ matrix, where each row and column represents a vertex in the graph. The diagonal entry is equal to the degree of the associated vertex. An off-diagonal entry is -1 , if the associated vertices of the row and column are connected in the graph, and 0 otherwise. Formally, let $d_{i}$ denote the degree of vertex $v_{i}$, and let $l_{i j}$ denote the entry of the Laplacian matrix at the $i$-th row and the $j$-th column, which we define as follows.

$$
l_{i j}= \begin{cases}d_{i} & \text { if } i=j \\ -1 & \text { if } \quad\left(v_{i}, v_{j}\right) \in E \quad \text { or } \quad\left(v_{j}, v_{i}\right) \in E \\ 0 & \text { otherwise }\end{cases}
$$

The Laplacian of the graph in Fig. 2.1 is

$$
L=\left(\begin{array}{rrrrrr}
2 & -1 & -1 & & & \\
-1 & 4 & -1 & -1 & -1 & \\
-1 & -1 & 3 & & -1 & \\
& -1 & & 2 & & -1 \\
& -1 & -1 & & 3 & -1 \\
& & & -1 & -1 & 2
\end{array}\right)
$$

We note that $L$ can also be defined as

$$
L=A^{T} A,
$$

where $A$ is the node-arc incidence matrix of the graph. This property holds regardless of the directions of edges in $G$. It is possible to add edge weights to the definition of Laplacian of a graph. In this case, the diagonal entry becomes the sum of weights of edges adjacent to the respective vertex, as opposed to the degree of this vertex, and the negative of the edge weight replaces "-1" as the off-diagonal entries. In this case, Eq. (2.1) can be rephrased as

$$
L_{w}=A^{T} D_{w} A,
$$

where $D_{w}$ is a diagonal matrix so that the $i$-th diagonal is the weight of edge $e_{i}$, and $L_{w}$ is the weighted Laplacian. Observe that a zero diagonal entry on $D_{w}$ corresponds to removing a line from the graph. 
The Laplacian of a graph is the basic element of spectral graph theory. Let $\lambda_{0} \leq \lambda_{1} \leq \ldots \leq \lambda_{n-1}$ be the eigenvalues of $L$. The Laplacian matrix is symmetric and semi-definite, and thus all eigenvalues are real and nonnegative. It is easy to see that $\lambda_{0}=0$, since all rows and columns of $L$ add up to zero, and thus the vector, $e$, whose entries are all the same and nonzero, is a singular vector for $L$. The smallest nontrivial eigenvalue $\lambda_{1}$ is more interesting due to its applications. Fiedler called $\lambda_{1}$ the algebraic connectivity of $G$ [13], as it provides a metric for the connectedness of a graph. If the graph inherently involves two loosely coupled sub-graphs, then $\lambda_{1}$ will be small. Fiedler also proved that $\lambda_{1}$ will decrease as we remove edges from the original graph, and it will be zero when the graph is decoupled into two disconnected components. A fundamental result in spectral graph theory generalizes this observation so that the multiplicity of the eigenvalue 0 gives the number of connected components in $G$.

Lemma 2.1. Let $L$ be the Laplacian of graph $G$, and let $\lambda_{0} \leq \lambda_{1} \leq \ldots \leq \lambda_{n-1}$ be its eigenvalues. If $\lambda_{i}=0$ and $\lambda_{i+1} \neq 0$, then $G$ has exactly $i+1$ connected components.

The multiplicity of eigenvalue 0 determines the number of connected components in a graph, associated eigenvectors identify these connected components. For an eigenvalue $\lambda_{i}=0$, the corresponding eigenvector $v_{i}$ has the same value for all vertices in a component, and a different value for each one of the $i+1$ components. This result underlies our analysis of the structure of an optimal solution in Section 4.2.3.

3. Problem formulation. Our focus in this work is to identify simple events that can trigger a cascading event, not to analyze consequences of cascading. Cascading events start with a significant disturbance to the system, and continue with failures of other system components, as these components are pushed beyond their capabilities, while the system is trying to avert a blackout. It will be the initial significant disturbance that we seek in this work, and thus we focus on static power flow analysis. Below, we first describe our power flow model and then describe how we measure the significance of an event. Finally, we cast the power grid vulnerability problem as a mixed integer nonlinear programming (MINLP) problem. In [11], a similar formulation is presented for a full power flow model with active and reactive power equations, and a slightly different load-shedding model.

3.1. Power system model. We consider a lossless power system network with $m$ buses (nodes) and $n$ lines (edges). We assume the voltages at the buses are fixed, and thus the dependence of real power injections at buses on the phase angle variables $\theta$ can be fully described by active power constraints, making the reactive power constraints unnecessary. The power flowing through the lines can be expressed as

$$
P_{\text {line }}=B \sin (A \theta),
$$

where $P_{\text {line }}$ is a vector of power flows over the lines, $B$ is a diagonal matrix whose diagonal entries correspond to line admittances, $A$ is a node-arc incidence matrix that represents the power network, and $\sin (A \theta)$ denotes a vector whose $i$-th component is $\sin \left((A \theta)_{i}\right)$. A vector of power injections $P$ is then obtained by adding the power flowing out of the buses into the network.

$$
A^{T} B \sin (A \theta)-P=0,
$$

with $A \theta$ taking values between $-\pi / 2$ and $\pi / 2$, as required for steady state stability.

Here, we will work with a given topology of the power grid and investigate the endurance of the grid to changes in topology. To extend the power flow equations 


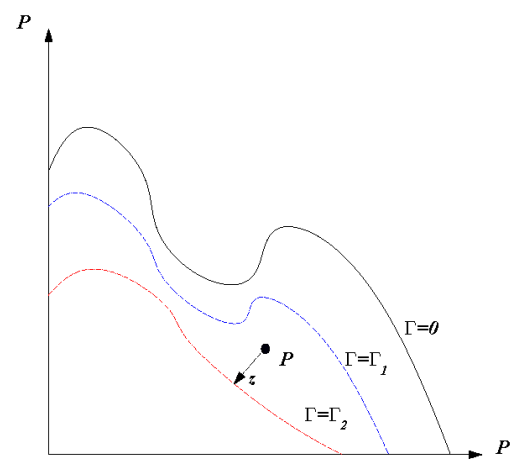

FIG. 3.1. Space of real power injections showing feasibility boundaries for various line statuses.

for changing topologies, we introduce binary-valued line parameters, $\gamma_{i}$ that indicate whether the $i$ th line is in service. That is,

$$
\gamma_{i}= \begin{cases}0 & \text { if the line is in service } \\ 1 & \text { if the line is out of service. }\end{cases}
$$

For simplicity of notation, we define $\Gamma=\operatorname{diag}(1-\gamma)$ as a diagonal matrix, whose $i$-th diagonal entry is $1-\operatorname{diag}(\gamma))$. The power flow model $(3.1)$, now with line parameters, can be expressed as

$$
F(\theta, P)=A^{T} B \Gamma \sin (A \theta)-P=0
$$

Removing lines from the network narrows the feasibility region of solutions to (3.2), as illustrated in Fig. 3.1. This figure shows the schematic view of (3.2) in $P$ space. When all lines under consideration are in service, the curve shown as a solid line represents a feasibility boundary for the power flow constraints. In the normal case, the system operating point lies inside the feasible region. When a line is removed from service, the feasibility boundary comes closer to the operating point, making the system more vulnerable to failure. Eventually, the removal of a line pushes the boundary past the operating point (dotted-lined curve), making system operation infeasible due to absence of a solution. This implies a blackout, and averting this blackout requires changing the loads and generation in the system, and hence moving the operating point $\mathrm{P}$, which we discuss in the next section.

3.2. Measuring the severity of a blackout. Load shedding means cutting off power supply to some loads when the demand becomes greater than the supply. While its common use is for high energy-demand times, broken power lines create subregions for which the demand cannot be met with the reduced transmission capability of the grid, even though supply is available in other parts of the system. Here, we use load shedding to minimally change load and generation to restore feasibility to the system to avert a blackout. From a mathematical point, a blackout corresponds to the current operating point, $P$, being outside of the feasibility region, and load shedding corresponds to finding the closest point to $P$ on the feasibility boundary, subject to some engineering constraints. The vector $Z$, which moves the operating point $P$ to the feasibility boundary, describes how to restore feasibility with minimum changes in loads, and the size of this vector can be used as an estimate of the size of a blackout. 
Suppose the nodes of the system are divided into two groups: generator nodes $N^{g}$ and load nodes $N^{l}$. For simplicity of presentation, we will reorder all the vectors and matrices so that generator nodes precede load nodes. Let $Z^{T}=\left(\left(Z^{g}\right)^{T},\left(Z^{l}\right)^{T}\right)$ be the vector that represents the change in power assignment to nodes, where $Z^{g}$ and $Z^{l}$ denote changes in generations and loads, respectively. By convention, $P_{i}^{l} \leq 0$ for all load nodes, and $P_{i}^{g} \geq 0$ for all generation nodes. This requires $Z_{i}^{g} \leq 0$ and $Z_{i}^{l} \geq 0$ for load shedding. An optimal load shedding strategy can be computed by solving the following optimization problem.

$$
\begin{array}{ll}
\min _{\theta, z} & -e^{T} Z^{g} \\
\text { s.t. } & F(\theta, P+Z)=0 \\
& P_{l} \leq P^{l}+Z^{l} \leq 0 \\
& 0 \leq P^{g}+Z^{g} \leq P^{g} \\
& -\pi / 2 \leq A \theta \leq \pi / 2
\end{array}
$$

Here, (3.4) corresponds to power flow equations. Constraints (3.5) and (3.6) guarantee that load and generation do not increase and remain as load and generation, respectively. The last constraint (3.7) ensures steady state stability. Since we assume a lossless system, the decrease in generation should match the decrease in consumption (i.e., $e^{T} Z^{g}=-e^{T} Z^{l}$ ), thus it is sufficient to look at only one of $Z^{g}$ and $Z^{l}$ to measure the total volume of load shed. Recall that $Z^{g}$ variables correspond to how much the generation will be cut, therefore they are negative, and thus we have the minus sign in the objective function, (3.3).

The Lagrangian $\mathcal{L}$ corresponding to (3.3)-(3.7) is

$$
\begin{aligned}
\mathcal{L} & =-e^{T} Z^{g}+\lambda^{T} F(\theta, P+Z)+\mu_{1}^{T}\left(-Z^{l}\right)+\mu_{2}^{T}\left(P^{l}+Z^{l}\right)+\mu_{3}^{T}\left(-P^{g}-Z^{g}\right) \\
& +\mu_{4}^{T}\left(Z^{g}\right)+\mu_{5}^{T}(-A \theta-\pi / 2)+\mu_{6}^{T}(A \theta-\pi / 2)
\end{aligned}
$$

where $\mu_{1}, \ldots, \mu_{6}$, and $\lambda$ are vectors of Lagrange multipliers. Karush-Kuhn-Tucker (KKT) conditions for the problem in (3.3)-(3.7) are as follows.

$$
\begin{aligned}
\left(\begin{array}{c}
-e \\
0
\end{array}\right)+\lambda^{T} \frac{\partial F}{\partial Z}+\left(\begin{array}{c}
\mu_{4}-\mu_{3} \\
\mu_{2}-\mu_{1}
\end{array}\right) & =0 \\
\lambda^{T} \frac{\partial F}{\partial \theta}+A^{T}\left(\mu_{6}-\mu_{5}\right) & =0 \\
\mu_{1} \cdot\left(-Z^{l}\right) & =0 \\
\mu_{2} \cdot\left(P^{l}+Z^{l}\right) & =0 \\
\mu_{3} \cdot\left(-P^{g}-Z^{g}\right) & =0 \\
\mu_{4} \cdot Z^{g} & =0 \\
\mu_{5} \cdot(-\pi / 2-A \theta) & =0 \\
\mu_{6} \cdot(A \theta-\pi / 2) & =0 \\
\mu_{1}, \ldots, \mu_{6} & \geq 0
\end{aligned}
$$

The notation "." in (3.11)-(3.16) is used to indicate component-wise multiplication of associated vectors. (3.9) and (3.10) correspond to the partial derivatives of $\mathcal{L}$ with respect to $Z$ and $\theta$, respectively, and equations (3.11)-(3.16) correspond to inequality 
constrainsts (3.5)-(3.7). Optimal solutions to problem (3.3)-(3.7) satisfy the KKT conditions (3.9)-(3.17). Thus the vector $Z$ that provides the best load shedding solution is obtained by solving equations (3.4) and (3.9)-(3.16), while honoring inequalities (3.5)-(3.7) and (3.17).

3.3. Power network vulnerability analysis problem as a MINLP. The power network vulnerability analysis problem can be described as the following MINLP problem.

$$
\begin{array}{cl}
\min _{\theta, \gamma, z} & e^{T} \gamma \\
\text { s.t. } & A^{T} B \Gamma \sin (A \theta)-(P+Z)=0 \\
& -\pi / 2 \leq A \Gamma \theta \leq \pi / 2 \\
& P_{l} \leq P^{l}+Z^{l} \leq 0 \\
& 0 \leq P^{g}+Z^{g} \leq P_{g} \\
& -e^{T} Z^{g} \geq S \\
& \left(\begin{array}{c}
-e \\
0
\end{array}\right)-\lambda+\left(\begin{array}{c}
\mu_{4}-\mu_{3} \\
\mu_{2}-\mu_{1}
\end{array}\right)=0 \\
& J \lambda+A^{T} \Gamma\left(\mu_{6}-\mu_{5}\right)=0 \\
& \mu_{1} \cdot\left(-Z^{l}\right)=0 \\
& \mu_{2} \cdot\left(P^{l}+Z^{l}\right)=0 \\
& \mu_{3} \cdot\left(-P^{g}-Z^{g}\right)=0 \\
& \mu_{4} \cdot Z^{g}=0 \\
& \mu_{5} \cdot(\pi / 2+A \Gamma \theta)=0 \\
& \mu_{6} \cdot(A \Gamma \theta-\pi / 2)=0 \\
& \mu_{1}, \ldots, \mu_{6} \geq 0 \\
& \gamma_{i} \in\{0,1\} \quad \text { for } \quad i=1,2, \ldots, m
\end{array}
$$

Here, (3.19) and (3.20) guarantee that there is a feasible solution to the power flow equations at $P+Z$, and (3.23) enforces that the resulting blackout is no smaller than a specified severity, $S$. Inequalities (3.21) and (3.22) are the load shedding constraints, and (3.24)-(3.32) correspond to the KKT conditions, so that $Z$ satisfies the necessary conditions for an optimal solution for the load shedding problem. In these equations, we have substituted $\frac{\partial F}{\partial Z}=I$, and $J=\frac{\partial F}{\partial \theta}$. Finally, (3.33) correspond to discrete line parameters, which indicate whether a line is cut $\left(\gamma_{i}=1\right)$ or active $\left(\gamma_{i}=0\right)$.

In this formulation, we are looking for the minimum number of lines to cut that will cause a disturbance no smaller than a specified severity. By switching the roles of the objective function (3.18) and the severity constraint (3.23), we can seek the maximum disturbance that can be created by cutting no more than a specified number of lines.

Our formulation reduces the problem to a MINLP problem. While recent efforts address solution strategies, MINLP problems are inherently hard to solve. Next, we will analyze the structure of an optimal solution to the problem (3.18)-(3.33) to approximate it as a pure combinatorial problem, with a lower complexity, both in theory and in practice. 
4. Reduction to a combinatorial problem. In this section, we analyze the structure of a feasible solution to our MINLP formulation to reveal a special combinatorial structure that can be exploited to approximate our MINLP formulation with a pure combinatorial problem. Our reduction enables us to directly seek the values of binary variables in the problem, without solving the nonlinear equations.

4.1. Structure of the Jacobian of the power flow equations. The Jacobian, $J$, of power flow equations in (3.2) with respect to $\theta$ is

$$
J=\frac{\partial F}{\partial \theta}=A^{T} B \Gamma \operatorname{diag}(\cos (A \theta)) A,
$$

where $\operatorname{diag}(\cos (A \theta))$ and $\Gamma$ are diagonal matrices whose $i$-th entries are equal to $\cos \left((A \theta)_{i}\right)$ and $1-\gamma_{i}$, respectively. Observe that $B, \Gamma$, and $\operatorname{diag}(\cos (A \theta))$ are all diagonal matrices with nonnegative diagonal entries, since $\gamma$ is a vector of binary variables and the angular differences represented by $A \theta$ are in the $[-\pi / 2, \pi / 2]$ range. Thus the Jacobian is identical in structure to a weighted Laplacian in (2.2).

When the diagonal entries in $B \Gamma \operatorname{diag}(\cos (A \theta))$ are all nonzero, $J$ has only a single zero eigenvalue, which means the network under consideration is initially connected. It is worth noting that when the power flow equation for a reference bus is removed from (3.2), along with its variable $\theta$, the resulting reduced order Jacobian does not have a zero eigenvalue, and is singular only when the operating point lies on the feasibility boundary [2]. We preserve the network structure by retaining the reference bus in order to be able to draw direct analogies with spectral graph theory. In our formulation, the Jacobian, $J$, is always singular with a single, trivial zero eigenvalue and the corresponding eigenvector $e=[1,1, \ldots, 1]^{T}$.

Recall from Section 2 that removal of an edge appears in the weighted Laplacian as a zero weight assignment to this edge. In the Jacobian, $J$ in (4.1), this happens when $\gamma_{i}=1$, which corresponds to removal of a line from the network, or when the angular difference for a line is $\mp \pi / 2$, which corresponds to capacity of a line being fully utilized. The multiplicity of eigenvalue zero is greater than one when the operating point lies on the feasibility boundary. From spectral graph theory, we know that a zero eigenvalue of $J$ with multiplicity greater than one means that the graph is fragmented into subgraphs, and further, the nodes in each subgraph can be discerned from the eigenvectors corresponding to the zero eigenvalue, as discussed in Section 2.

The power flow Jacobian, $J$, is analogous to the residual graph for flow problems in graph theory, which represents the incremental transmission capability of the network. In a residual graph, edge capacities correspond to unused edge capacities, and the incremental transmission capability of the system is measured by the total flow capacity from the source to the terminal in the residual graph. At an optimal solution (i.e. the maximum flow), the source and the terminal are disconnected in the residual graph, corresponding to zero incremental transmission capability. In power systems, the flow between two nodes is determined by the sine of the angular difference between the two nodes, thus the cosine of this angular difference can be viewed as the residual capacity of this line. When the operating point is on the boundary of feasibility, the incremental transmission capability of the system will cease, which is reflected by the multiplicity of the zero eigenvalue being more than 1. By Lemma 2.1, the system is divided into at least two subgroups that are connected by either saturated lines with angular difference at $\mp \pi / 2$ or removed lines due to the $\gamma$ variables.

4.2. Structure of a feasible solution. An analysis of the Lagrangian multipliers sheds light onto the structure of a solution for problem (3.18)-(3.33). We will 
show that the system is decomposed into a generation-rich region and a load-rich region. Then, we will study the flow on the lines between these regions.

4.2.1. Decomposition into load and generation-rich regions. Let $\lambda=$ $\left(\lambda^{g^{T}}, \lambda^{l^{T}}\right)^{T}$ be partitioned into variables for generator and load nodes, so that we can split (3.24) into two equations.

$$
\begin{array}{r}
-e-\lambda^{g}+\mu_{4}-\mu_{3}=0 \\
0-\lambda^{l}+\mu_{2}-\mu_{1}=0
\end{array}
$$

Consider a generator vertex and the associated Lagrangian multiplier $\lambda_{i}^{g}$. If $\lambda_{i}^{g}<-1$ then by (4.2), the corresponding $\mu_{3}$ variable must be positive. This requires $Z_{i}^{g}+P_{i}^{g}=$ 0 by (3.28), which means the generation at this node will be zero after load shedding. If $\lambda_{i}^{g}=-1$, then the corresponding $\mu_{3}$ and $\mu_{4}$ variables must be equal by (4.2). This is only possible when they are both zero, since $\mu_{3}$ and $\mu_{4}$ correspond to lower and upper bounds on $P^{g}+Z^{g}$. In this case, neither bound is binding and the generation after load shedding is anything in the range $\left[0, P_{i}^{g}\right]$. Finally, if $\lambda_{i}^{g}>-1$ then by (4.2), the corresponding $\mu_{4}$ variable must be positive. This requires $Z_{i}^{g}=0$ by (3.28), which means that there will be no decrease in the generation at this node.

We can do a similar analysis for the load nodes. Consider a load vertex and the associated Lagrangian multiplier $\lambda_{i}^{l}$. If $\lambda_{i}^{l}>0$ then by (4.3), the corresponding $\mu_{2}$ variable must be positive. This requires $Z_{i}^{l}+P_{i}^{l}=0$ by (3.27), which means the load at this node will be zero after load shedding. If $\lambda_{i}^{l}=0$, then the corresponding $\mu_{1}$ and $\mu_{2}$ variables must be equal by (4.3). This is only possible when they are both zero, since $\mu_{1}$ and $\mu_{2}$ correspond to lower and upper bounds on $P^{l}+Z^{l}$. In this case, neither bound is binding and the load after shedding is anything in the range $\left[0, P_{i}^{l}\right]$. Finally, if $\lambda_{i}^{l}<0$ then by (4.3), the corresponding $\mu_{1}$ variable must be positive. This requires $Z_{i}^{l}=0$ by (3.28), which means that there will be no decrease in the load at this node.

This yields the following load-shedding model. For generation nodes,

$$
\begin{aligned}
& Z_{i}^{g}=0 \quad \text { if } \quad \lambda_{i}>-1, \\
& 0 \leq Z^{g}+P^{g} \leq P^{g} \quad \text { if } \quad \lambda_{i}=-1, \\
& Z_{i}^{g}=-P_{i}^{g} \quad \text { if } \quad \lambda_{i}<-1 .
\end{aligned}
$$

And for loads

$$
\begin{array}{rll}
Z_{i}^{l}=0 & \text { if } & \lambda_{i}<0, \\
P^{l} \leq Z^{l}+P^{l} \leq 0 & \text { if } & \lambda_{i}=0, \\
Z_{i}^{l}=-P_{i}^{l} & \text { if } & \lambda_{i}>0 .
\end{array}
$$

Observe that not all $\lambda_{i} \geq 0$, since that requires $Z^{g}=0$ for all generation nodes, which contradicts the blackout severity constraint, $-e^{t} Z^{g} \geq S$. Similarly, not all $\lambda_{i}<0$, since that requires $Z^{l}=0$ for all loads. Since we have a lossless system, $Z^{l}=0$ implies, $Z^{g}=0$, which again contradicts the blackout severity constraint.

Based on these observations, we can decompose the system into two regions based on their Lagrangian multipliers. Let the first group be composed of nodes for which $\lambda_{i}<0$, and the second group be composed of the remainder for which $\lambda_{i} \geq 0$. For the first region $\left(\lambda_{i}<0\right)$, we know by (4.7) that the loads cannot be decreased while the generation can be decreased as necessary. For the second region $\left(\lambda_{i} \geq 0\right)$, we know by 
(4.4) that the generation cannot be decreased, whereas the loads can be decreased as necessary. Thus the Lagrangian multipliers give a decomposition of the system into two regions: a generation-rich region, defined by $\lambda_{i}<0$, where only the generation can be decreased and loads remain the same, and load-rich region, defined by $\lambda_{i} \geq 0$, where only the loads can be decreased and the generation remains the same.

This shows that the $\lambda$ vector decomposes the system into a generation-rich part $P_{1}$ and a load-rich part $P_{2}$. The reason for the blackout is the failure to transmit power from the generation-rich part to the load-rich part, and moreover, the best way to restore the system to feasibility is to decrease the generation in the generation-rich part and the load in the load-rich part.

4.2.2. Flow between the two regions. In the previous section, we showed that at a feasible solution to (3.18)-(3.33), the system will be decomposed into a generation-rich region, $P_{1}$ and a load-rich region, $P_{2}$. Now, we study the flow between these two regions by investigating (3.25), and show that there is at least one line between the two regions that uses its maximum capacity to carry power from the generation-rich side to the load-rich side.

For simplicity of presentation, we assume all vectors are permuted so that the nodes in the generation-rich region are ordered before those in the load-rich region, and the same permutation is applied to matrices symmetrically. Let $\lambda=\left(\lambda^{1^{T}}, \lambda^{2^{T}}\right)^{T}$, so that $\lambda^{1}$ corresponds to the nodes in the generation-rich region $\left(\lambda_{i}^{1}<0\right)$, and $\lambda^{2}$ corresponds to the nodes in the load-rich region $\left(\lambda_{i}^{2} \geq 0\right)$. Then (3.25) can be rewritten as

$$
\left(\begin{array}{ll}
J_{11} & J_{12} \\
J_{21} & J_{22}
\end{array}\right)\left(\begin{array}{c}
\lambda_{1} \\
\lambda_{2}
\end{array}\right)+A^{T} \Gamma\left(\mu_{6}-\mu_{5}\right)=0
$$

where $J_{11}, J_{12}, J_{21}$, and $J_{22}$ are submatrices of $J$ that conform with $\left(\lambda^{1^{T}}, \lambda^{2^{T}}\right)^{T}$. Recall that $J$ is symmetric and diagonally dominant, with the only positive entries on the diagonals, and the sum of absolute values of the remaining entries on a column/row is equal to the diagonal entry. Thus, $e^{T} J_{11} \lambda^{1} \leq 0$, where the equality is satisfied only when $J_{21}=M_{0}$, where we use $M_{0}$ to denote a matrix of all zeros. Similarly, $e^{T} J_{22} \lambda^{2} \geq 0$, where the equality is satisfied only when $J_{12}=M_{0}$. Observe that $J_{12}$ and $J_{21}$ matrices correspond to the lines between the two regions, and their nonzeros correspond to lines that are neither cut $\left(\gamma_{i}=1\right)$ nor saturated due to the angular difference being $\mp \pi / 2$. Therefore, $J_{12}=J_{21}=0$ means that all lines

In general, when $J_{12}=J_{21}^{T} \neq 0$, which means there may be unsaturated lines between the two regions. In this case,

$$
e^{T}\left(J_{11}, J_{12}\right)\left(\lambda^{1^{T}}, \lambda^{2^{T}}\right)^{T}<0,
$$

since $e^{T} J_{11} \lambda^{1}<0, J_{12}$ is composed of all negative entries, and $\lambda^{2}$ is composed of all nonnegative entries. For similar reasons,

$$
e^{T}\left(J_{21}, J_{22}\right)\left(\lambda^{1^{T}}, \lambda^{2^{T}}\right)^{T}>0 .
$$

This means for (4.10) to be satisfied, $A^{T} \Gamma\left(\mu_{6}-\mu_{5}\right) \neq 0$. The Lagrangian multipliers $\mu_{5}$ and $\mu_{6}$ are for lower and upper bounds on $A \theta$, and thus only one can be nonzero, when the anguler difference at the corresponding line is $\mp \pi / 2$. Thus there must be at least one active line $\left(\gamma_{i}=0\right)$ that is saturated. Recall that each column of $A^{T}$ has one " -1 " and one " 1 ", and thus the saturated line that is internal in one of the regions will not help, and we need a saturated line that goes between the two regions. 
Assume this line is directed from $P_{1}$ to $P_{2}$, that is the column in $A^{T}$ for this line has its -1, in the generation-rich part, and its 1 in the load-rich part. This implies that the corresponding entry in $\mu_{5}$ needs to be positive, since we need a positive addition to the first part. The Lagrangian multiplier $\mu_{5}$ can be positive only when the angular difference for this line is $-\pi / 2$, which means the power flows from the generation-rich side $P_{1}$ to the load-rich side $P_{2}$. Symmetrically, if the line is directed from $P_{2}$ to $P_{1}$ in matrix $A^{T}$, then $\mu_{6}$ needs to be positive, which means angular difference for this line is $\pi / 2$, and thus power flows again from the generation-rich side to the load-rich side. This observation does not hold for each line on the boundary, but we know that it holds for at least one boundary line.

4.2.3. Analysis of a special case. In this section we analyze a special case where the Lagrangian multipliers for the constraints on angular differences are set to be zero, i.e., $\mu_{5}=\mu_{6}=0$. This corresponds to a degenerate case, and our goal here is to better disclose the combinatorial structure in a solution to (3.18)-(3.33). While our results in the remainder of the paper do not rely on the results in this section, we believe what we present can play an important enabling role for future studies on the topic.

The Lagrangian multipliers $\mu_{5}$ and $\mu_{6}$ being zero reduces (3.25) to $J \lambda=0$. As shown in Section 4.2.1, all entries of $\lambda$ cannot be the same, and some of them need to be nonnegative, and some need to be negative. This excludes the $\lambda=(1,1, \ldots, 1)^{T}$, solution, thus we need another singular vector for $J$. By our discussions in Section 2, we know that for $J$ to have another singular vector the graph corresponding to $J$ should be decomposed into multiple components, which is possible due to broken and saturated lines. This means in a solution to our problem, the power grid will be decomposed into at least two groups, so that the edges connecting these groups are either cut $\left(\gamma_{i}=1\right)$, or saturated (the angular difference is $\left.\mp \pi / 2\right)$. For brevity, we assume there are exactly two groups in the system.

The entries in the associated singular vector $\lambda$ will reflect this decomposition of the grid and each $\lambda_{i}$ will be assigned one of the two real numbers, $c_{1}$ and $c_{2}$. Let $c_{1}<c_{2}$, and let $P_{1}$ be the set of nodes for which $\lambda_{i}=c_{1}$ and $P_{2}$ be the set of remaining nodes, for which $\lambda_{i}=c_{2}$. In Section 4.2.1 we showed that there must be some generators, for which $\lambda_{i}^{g} \leq-1$, and there must be some loads, for which $\lambda_{i}^{l} \geq 0$. Assume $c_{1}=-1$ and $c_{2}=0$. Note that this choice does not constrain the other variables, and any other solution can only be as good as a solution with $c_{1}=-1$ and $c_{2}=0$. The load shedding model in (4.4)-(4.9) still applies, which means that a feasible solution does not decrease the loads on the $P_{1}$ nodes, and does not decrease the generation on the $P_{2}$ nodes. This shows that the $\lambda$ vector decomposes the system into a generation-rich part $P_{1}$ and a load-rich part $P_{2}$, as discussed before. The reason for the blackout is the failure to transmit power from the generation-rich part to the load-rich part, since all lines between these two parts are either cut, or already operating at their maximum limits. Moreover, the best way to restore the system to feasibility is to decrease the generation in the generation-rich part, and the load in the load rich part.

4.3. Power network vulnerability analysis as a combinatorial problem. While the problem (3.18)-(3.33) provides an accurate mathematical formulation for vulnerability analysis, its solution provides more than a small set of critical lines. A solution to the problem (3.18)-(3.33) computes how load can be shed optimally, phase angles at nodes after load shedding, and a decomposition of the system into load and generation-rich regions. What we really need is only the set of broken lines, i.e, the vector $\gamma$. We need to know the norm of the $Z$ vector is above a specified severity 
threshold, but we don't need to know its entries individually. Note that once $\gamma$ is known, computing the remaining information in the formulation only requires solving a system of nonlinear equations.

Optimal load shedding requires decreasing the loads in the load-rich region, while keeping the generation as is. Symetrically, we need to cut the generation in the generation-rich region, and retain the loads. The total volume to be shed is defined by the load-generation mismatch in a part, and the total flow on the active boundary edges between the two parts. In this model, the blackout severity is a function of the decomposition of the system and depends on the load generation mismatch in the two regions. Therefore, constraint (3.23) can be satisfied with the right choice of partitioning. Let $T$ denote the total power being transmitted from one part to another in the remaining network after lines are removed. Then $-e^{T} Z^{g}$ can be computed as

$$
\left(\sum_{\lambda_{i}<0} P_{i}\right)-T
$$

Here, the summation computes the excess generation in $P_{1}$, and since we cannot cut the loads in this part, the generation must be reduced to match the load after the lines leaving this part are loaded maximally.

We know that at a feasible solution, the power grid will be decomposed into two parts as a load-rich region and a generation-rich region. What (4.11) shows is that an optimal solution seeks for a decomposition that maximizes the generation/load mismatch and minimizes the potential power transmission between the two regions, which reveals a clear combinatorial structure in the problem. This raises an interesting question of whether we can solve problem (3.18)-(3.33), by directly looking for such a decomposition. Below, we discuss how such a decomposition can be used to find an approximation to the MINLP formulation and why this is a good approximation. The big gain here is that the decomposition problem can be formulated as a MILP, as opposed to a MINLP. It should be noted that our reduction is more than merely solving the discrete portion of a MINLP problem in a decomposition algorithm such Benders decomposition or outer approximation. Our formulation foresees the change in the nonlinear part, and directly seeks values of discrete variables in an optimal solution to the MINLP, without explicitly solving the nonlinear part.

For a formal definition, let Lines $\left(P_{1}, P_{2}\right)$ denote the set of lines between parts $P_{1}$ and $P_{2}, \operatorname{Cap}(E)$ be the total capacity of lines in set $E$, and $L\left(P_{1}\right)$ and $G\left(P_{1}\right)$ be the total load and generation in part $P_{1}$, respectively. We define the network vulnerability analysis problem as follows.

Let $G=(V, E)$ be a graph with $V$ as the set of nodes (buses), and $E$ as the set of edges (lines), and let $S$ be a specified severity threshold. Find a minimum cardinality subset of edges $C \subseteq E$, so that there exists a partitioning of nodes $V$ into $P_{1}$ and $P_{2}=V \backslash P_{1}$, so that

$$
G\left(P_{1}\right)-L\left(P_{1}\right)-\operatorname{Cap}\left(\operatorname{Lines}\left(P_{1}, P_{2}\right) \backslash C\right) \mid \geq S .
$$

This problem can be solved as the network inhibition problem in graph theory [22], which we will address in the next section. There are two reasons for why the combinatorial model is an approximation, and not an exact model. Both reasons cause underestimation of the severity of a blackout, and thus a solution to the combinatorial problem will yield a feasible solution to (3.18)-(3.33), but not necessarily an optimal one. 
Firstly, we know that an optimal solution involves a decomposition of the system, where only the generation (load) will be shed in the generation (load)-rich region, but we do not know if this holds for an arbitrary decomposition. That is in an optimal solution, it is sufficient to shed the load or generation to merely match the other, but this is not necessarily the case for all decompositions, as we might have to lower the generation even in the generation rich region to restore feasibility. This will cause underestimating the blackout severity, as our combinatorial model does not fully capture the complexity of power flow equations. However, this is not as serious a drawback, since we are only looking for significant blackouts and will cut a significant portion of the generation and loads. This translates to a large feasible space for the minimal load shedding problem, within which it is possible to find an instance where only cutting the loads or generation is sufficient.

The second reason why the combinatorial model is not exact is that the total flow between the two parts may be less than the cumulative capacities of the connecting edges. We know that there will be at least one line that uses its maximum capacity to transfer power from the generation-rich side to the load-rich side. And in our analysis of a special case in Section 4.2.3, we showed that all lines between the two parts will be saturated. While the cumulative capacity of lines is not always utilized for a given decomposition of the system, the particular decomposition we choose creates a load/generation mismatch, and minimizing the total volume of load shedding requires maximizing the total flow from the generation-rich side to the load-rich side. Thus the goal of load shedding can be considered as maximizing the flow between the two regions. Therefore, what we use as an approximation is an upper bound on the value of a maximization problem that is implicit in the KKT conditions in (3.18)-(3.33), and thus a good approximation.

5. Solving the network inhibition problem. In the network inhibition problem, we aim to find the best way to attack a network to minimize its transmission capability. In graph theoretical terms, the network inhibition problem tries to find the most cost-efficient subset of lines, removal of which minimizes the maximum flow on the remaining network. The network inhibition problem naturally involves the maximum flow problem as a subproblem. Below, we first discuss flow networks, and define the network inhibition problem. Then we provide an integer programming formulation for this problem, and discuss how the power network vulnerability analysis problem can be posed as the network inhibition problem.

5.1. Flow graphs and the maximum flow problem. A flow network $G=$ $(V, E)$ is defined by a set of vertices $V$, a set of edges $E$, where each edge $(u, v)$ has a nonnegative capacity $c(u, v)$, and two special vertices: a source $s$ and a terminal $t$. A flow in $G$ is a real valued function, $f: E \rightarrow \mathcal{R}$. We use $f(u, v)$ to refer to a flow on the edge from vertex $u$ to vertex $v$. Using a single source and a single terminal vertex provides a standard form for the maximum flow problem, and even if there are multiple vertices with production, a single source vertex, $s$, is used, which is connected to all other vertices with production, and the capacity of the connecting edge is equal to the production on that node. Similarly, only a single terminal vertex, $t$, is used, which is connected to all other vertices with consumption, and the capacity of the connecting edge is equal to the consumption on that node. We say a flow is feasible if it respects conservation of flow and the capacity constraints on edges. Conservation of flow requires that the total flow into a node is equal to total flow out of that node except for the source and terminal vertices. The value of a flow is defined by the total flow leaving the source, and the maximum flow problem aims to find a feasible flow 


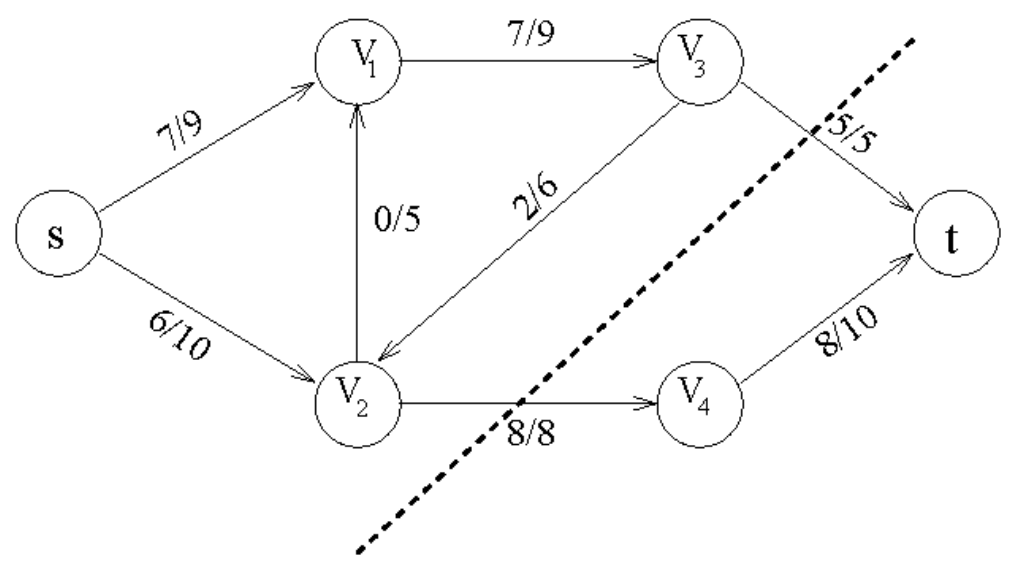

FIG. 5.1. Maximum-flow and minimum cut on a flow graph. Numbers on the edges represent flow assignment/capacity. The dashed line represents the cut.

with maximum value.

A closely related concept to maximum flow is the minimum cut. A cut in a flow graph is defined by a bipartitioning of vertices $V$ into $S$ and $T=V \backslash S$, so that $s \in S$ and $t \in T$. We say an edge is on the cut if one of its end vertices is in $S$ and the other is in $T$. The capacity of a cut is defined as the sum of capacities of the edges on the cut, and a minimum cut is one with minimum capacity among all the cuts. It is easy to see that the capacity of any cut is an upper bound on the value of a maximum flow, since the edges on the cut block all paths from the source to the terminal, and thus the total flow cannot exceed their cumulative capacity. As one of the earliest and fundamental results in combinatorial algorithms, Ford and Fulkerson proved that the capacity of a minimum cut is equal to the value of a maximum flow. This duality between maximum flow and minimum cut underlies many algorithms for flow problems and in this work we compute the value of a maximum flow by finding the capacity of a minimum cut. A more detailed discussion on flow algorithms along with proofs of this duality can be found in $[7,26]$.

An example for the maximum flow minimum cut property is illustrated in Fig. 5.1. In this figure, the numbers on edges represent the flow assignment and the capacity of the edge. For instance, the edge from $v_{1}$ to $v_{3}$ has a capacity of 9 units and uses 7 units of this capacity in the current flow assignment. The volume of a maximum flow in this graph is 13 units, and the current flow assignment is optimal. The associated minimum cut in this graph is $S=\left\{s, v_{1}, v_{2}, v_{3}\right\}$ and $T=\left\{v_{4}, t\right\}$ with capacity 13 .

5.2. Network inhibition problem. In [22], Phillips defines the network inhibition problem as follows. Each edge in the network has a destruction cost, and a fixed budget is given to attack the network. A feasible attack removes a subset of the edges, whose total destruction cost is no greater than the budget, and the network inhibition problem is to find an attack that optimally reduces the value of a maximum flow in the graph after the attack. The network inhibition problem has two objectives and/or constraints: the cost of an attack and the resulting damage. Phillips' formulation, which we call the maximum damage version of the network inhibition problem, constrains the budget of the attack and seeks to maximize the damage. Here, we work on the minimum cost version of the problem, where we look for the most cost-effective 
attack, where the damage is no smaller that a specified bound.

The network inhibition problem is closely related to the maximum flow/minimum cut problem. More specifically, the minimum cut problem is a special version of the network inhibition problem, where the value of a maximum flow in the network after the attack should be zero. In terms of complexity however, the network inhibition problem is much harder. While maximum flow problems can be efficiently solved by polynomial-time algorithms [14], the network inhibition problem is NP-complete [22]. Phillips provides a comprehensive study on the network inhibition problem [22]. Royset and Wood [24] studied this problem as a bi-objective problem, and Pinar et al. [23] studied the inhibiting bisection problem, where a graph decomposition with maximum production/demand mismatch is sought.

5.3. MILP formulation for the network inhibition problem. A formulation for the network inhibition problem requires measuring the value of a maximum flow on the graph after the attack, which we do by finding a minimum cut. For clarity of presentation, we first present an integer programming formulation of the minimum cut problem and then extend this formulation for the network inhibition problem.

5.3.1. MILP formulation for the minimum cut problem. Let $G=(V, E)$ be a flow network with $n$ vertices and $m$ edges, and let $A$ be the $m \times n$ node-arc incidence matrix of this graph. We assume the first and last columns of $A$ correspond to the source and terminal vertices, respectively. We use $c_{i}$ to refer to the capacity of the $i$-th line. We define a binary variable $\rho_{i}$ for each vertex $v_{i} \in V$, so that

$$
\rho_{i}=\left\{\begin{array}{ll}
0 & \text { if } v_{i} \in S \\
1 & \text { if } v_{i} \in T
\end{array},\right.
$$

where $S$ and $T=V \backslash S$ denote the partitioning of $V$ that defines the cut. We also define a binary variable $\omega_{i}$ for each edge, so that

$$
\omega_{i}= \begin{cases}1 & \text { if } e_{i} \text { is on the cut } \\ 0 & \text { otherwise }\end{cases}
$$

The minimum cut problem can then be formulated as follows.

$$
\begin{array}{ll}
\min _{\rho, \omega} & c^{T} \omega \\
\text { s.t. } & A \rho-\omega \leq 0 \\
& A \rho+\omega \geq 0 \\
& \rho_{1}=0 \\
& \rho_{n}=1 ; \\
& \rho_{i} \in\{0,1\} \text { for } i=1,2, \ldots, n \\
& \omega_{i} \in\{0,1\} \text { for } i=1,2, \ldots, m
\end{array}
$$

Here, the objective function minimizes the cumulative capacity of the cut edges. Constraints (5.4) and (5.5) guarantee that $s \in S$ and $t \in T$, respectively. Constraints (5.6) and (5.7) guarantee that the $\rho$ and $\omega$ are binary variables. Constraints (5.2) and (5.3) are used to enforce any edge between parts $S$ and $T$ to be labeled as a cut edge. Consider an edge $e_{k}$ that goes from $v_{i}$ to $v_{j}$, for which we have the following constraints.

$$
\begin{aligned}
& \rho_{j}-\rho_{i}-\omega_{k} \leq 0 \\
& \rho_{j}-\rho_{i}+\omega_{k} \geq 0
\end{aligned}
$$


We need to show that $\omega_{k}=1$, if $v_{i}$ and $v_{j}$ are on different parts $\left(\rho_{i} \neq \rho_{j}\right)$. If $\rho_{j}=1$ and $\rho_{i}=0,(5.8)$ forces $\omega_{k}$ to be $\geq 1$. Symmetrically, if $\rho_{j}=0$ and $\rho_{i}=1$, it will be (5.9) that forces $\omega_{k}$ to be $\geq 1$. When the two vertices are in the same part, i.e., $\rho_{i}=\rho_{j}, \omega_{k}$ can be either zero or one. However, since the objective is to minimize $c^{T} \omega$, when the edge is not on the cut, $\omega_{k}$ will be at its minimum, zero. This analysis further shows that, we do not need to impose $\omega$ variables to be binary explicitly. When edge $e_{k}$ is on the cut, we need $\omega_{k} \geq 1$, and when $e_{k}$ is an internal edge, $\omega_{k}$ will be at its minimum due to the objective function. In an optimal solution to (5.1)-(5.6), $\omega$ variables naturally take binary values, when they are constrained to be in the $[0,1]$ region. Therefore we can replace (5.7) with

$$
0 \leq \omega_{i} \leq 1 \text { for } i=1,2, \ldots, m
$$

5.3.2. MILP formulation for the network inhibition problem. Since the network inhibition problem seeks to minimize the maximum flow/minimum cut of a graph in a cost optimal way, we can use our formulation for the minimum cut as the core of our formulation for the network inhibition problem. We start by defining a binary variable $d_{i}$ for each edge that defines whether a line is destroyed.

$$
d_{i}= \begin{cases}1 & \text { if } e_{i} \text { is destroyed } \\ 0 & \text { otherwise }\end{cases}
$$

Let $p$ be a vector that indicates line-destruction costs. The minimum cost version of the network inhibition problem can be formulated as a MILP problem as follows.

$$
\begin{array}{ll}
\min _{\rho, \omega} & p^{T} d \\
\text { s.t. } & c^{T} \omega \leq S \\
& A \rho-(\omega+d) \leq 0 \\
& A \rho+(\omega+d) \geq 0 \\
& \rho_{1}=0 \\
& \rho_{n}=1 ; \\
& \rho_{i} \in\{0,1\} \text { for } i=1,2, \ldots, n \\
& d_{i} \in\{0,1\} \text { for } i=1,2, \ldots, m \\
& \omega_{i} \in[0,1] \text { for } i=1,2, \ldots, m
\end{array}
$$

Here, the objective corresponds to minimizing the cost of the attack, and the $\omega$ vector identifies the cut edges on the remaining graph. While this cut is not necessarily the minimum cut, it provides an upper bound on the maximum flow in the graph, which is sufficient for our purposes. Inequality (5.11) guarantees the capacity of this cut, thus the volume of a maximum flow, is no bigger than a specified threshold $S$. Equations (5.12)-(5.15) ensure that the $\omega$ vector identifies the cut edges. The source and the terminal vertices are on different parts of the cut due to (5.14) and (5.15), and for each line $e_{k}=\left(v_{i}, v_{j}\right)$ in the graph we have the following two constraints.

$$
\begin{aligned}
& \rho_{j}-\rho_{i}-\omega_{k}-d_{k} \leq 0 \\
& \rho_{j}-\rho_{i}+\omega_{k}+d_{k} \geq 0
\end{aligned}
$$


It suffices to show that $\omega_{k}=1$, if $v_{i}$ and $v_{j}$ are on different parts $\left(\rho_{i} \neq \rho_{j}\right)$ and the edge is not destroyed $\left(d_{k}=0\right)$. Note that we do not need to show that $\omega_{k}=0$ for other cases, since $\omega_{k}>0$ redundantly increases the $c^{T} \omega$ value, which we are trying to keep small. In other words, the $\omega$ vector defines a valid cut in the graph, but it is not necessarily minimal, and a subset of the edges it provides, might provide a valid cut. If $\rho_{j}=0, \rho_{i}=1$, and $d_{i}=0,(5.19)$ forces $\omega_{k} \geq 1$. Symmetrically, if $\rho_{j}=1, \rho_{i}=0$, and $d_{i}=0$, then it will be (5.20) that forces $\omega_{k}$ to be $\geq 1$. Also observe that when $d_{i}=1$, the two inequalities will be satisfied, regardless of $\rho_{i}, \rho_{j}$ and $\omega_{k}$.

The formulation in (5.10)-(5.18) is the minimum cost version of the network inhibition problem, where we look for the most cost-efficient way to cause a damage of specified severity. We can easily switch the positions of the objection function and the severity constraint (5.11) for the maximum damage version of the problem, where we try to find an attack of specified cost that willgive the maximum damage.

5.4. Power grid vulnerability as a network inhibition problem. The combinatorial version of the vulnerability analysis problem of Section 4.3 can be posed as the network inhibition problem. Observe that the severity constraint, which we stated as the volume of load shed being above a threshold, $S$, can be rephrased as the remaining flow in the graph being below $|G|-S$, where $|G|$ denotes the total generation in the system. Here, we replace the severity threshold $S$ of the power grid vulnerability analysis with $|G|-S$ for the network inhibition problem. The graph of a power grid can be transformed to a flow graph by adding a source vertex $s$ and a terminal vertex $t$, and connecting each generator to the source with an edge whose capacity is equal to the generation at that node and connecting each load to the terminal with an edge whose capacity is equal to the consumption at that node. All other edges that correspond to power lines retain their capacities. We define the destruction cost of the source and terminal edges to be $\infty$, and all other edges to be 1 , to guarantee that the solution to the network inhibition only chooses actual power lines to cut.

A solution to the network inhibition problem identifies a bipartitioning of the nodes into $S$ and $T$, which correspond to generation-rich region $P_{1}$ and load-rich region $P_{2}$, respectively. The cut edges potentially includes source edges that connect $s$ to generation nodes, terminal edges that connect $t$ to terminal nodes, as well edges that represent power lines. Observe that a source edge will be cut, if the respective generator is in $T$, and similarly a terminal edge will be cut if the respective load is in $S$. The capacity of the cut can then be expressed as $L\left(P_{1}\right)+G\left(P_{2}\right)+\operatorname{Cap}\left(\operatorname{Lines}\left(P 1, P_{2}\right) \backslash\right.$ $C)$. Here $L\left(P_{1}\right)$ represents the total load in $S$ and is equal to the cumulative capacity of the terminal edges on the cut. Similarly, $G\left(P_{2}\right)$ represent the total generation in $T$ and is equal to cumulative capacity source edges on the cut. $C$ is the set of cut edges identified by the $d$ vector, and $\operatorname{Cap}\left(\operatorname{Lines}\left(P 1, P_{2}\right) \backslash C\right)$ is the total capacity of the active edges on the cut that represent power lines. Constraint (5.11) then becomes

$$
\begin{aligned}
L\left(P_{1}\right)+G\left(P_{2}\right)+C a p\left(\operatorname{Lines}\left(P 1, P_{2}\right) \backslash C\right) & \leq|G|-S \\
L\left(P_{1}\right)+|G|-G\left(P_{1}\right)+C \operatorname{Cap}\left(\operatorname{Lines}\left(P 1, P_{2}\right) \backslash C\right) & \leq|G|-S \\
L\left(P_{1}\right)-G\left(P_{1}\right)+C \operatorname{Cap}\left(\operatorname{Lines}\left(P 1, P_{2}\right) \backslash C\right) & \leq-S \\
-L\left(P_{1}\right)+G\left(P_{1}\right)-C a p\left(\operatorname{Lines}\left(P 1, P_{2}\right) \backslash C\right) & \geq S,
\end{aligned}
$$

which is the same as (4.12).

6. Experimental results. We present two sets of experiments to show that our proposed techniques are accurate and practical. The first set of experiments show that 


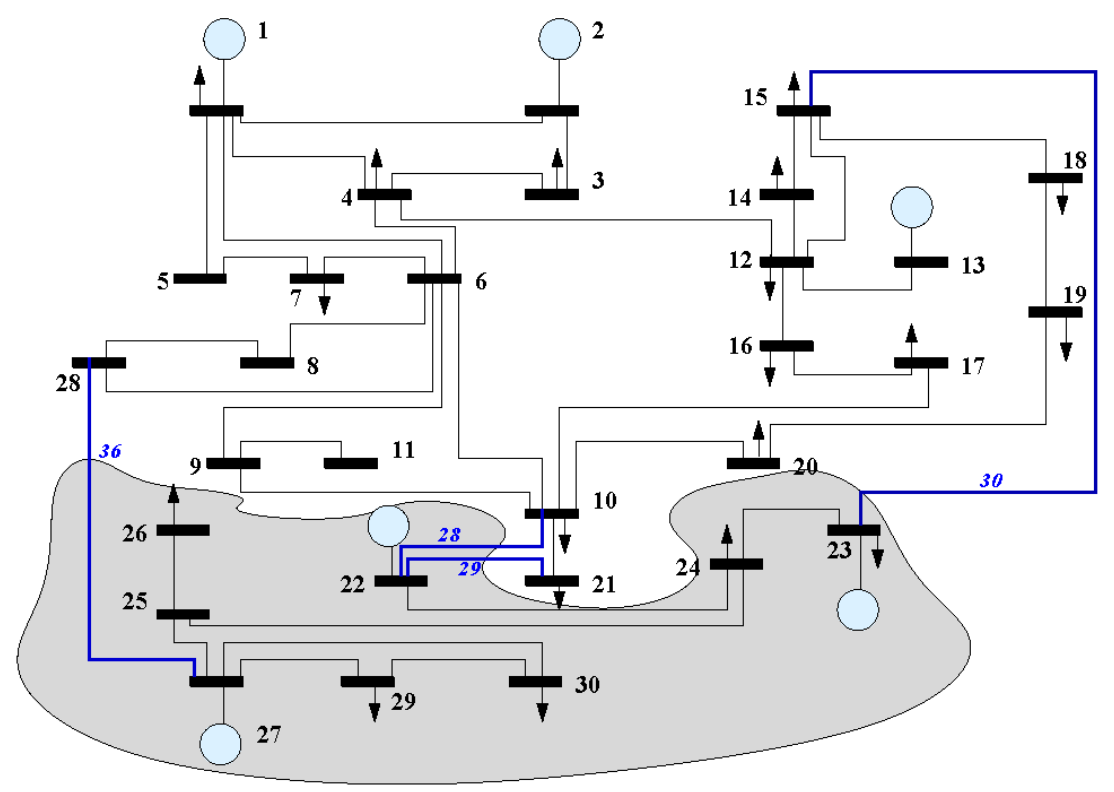

FIG. 6.1. IEEE 30-bus system

the gap between our combinatorial approximation and the original MINLP formulation is small and rapidly closes as the severity of the blackout increases. In the second set of experiments, we apply our integer programming formulation for the network inhibition problem to a simplified model of the Western power grid with 13,374 nodes and 16,520 lines to show the practicality of our simplified formulation.

For the first set of experiments, we used a slightly modified version of the IEEE 30 bus system [1] as described in [11], where the generator active power injections are modified so that there is no natural power balance in the system, providing a better test case for vulnerability analysis. This modified data set is presented in detail in [11], and the IEEE 30 bus system is illustrated in Fig. 6.1.

In this system, the generation-rich lower subsystem (the shaded region in Fig. 6.1) is connected to the load-rich upper subsytem with only 4 lines (lines 28, 29, 30, and 36). Failures among these lines can cause a blackout, as the remaining lines are not sufficient to transfer power from the generation-rich subregion to the load-rich region. In our experiments, we have looked at 5 different combinations of line failures, as lines 28, 29, and 30, lines 28, 29, and 36, lines 28 and 29, lines 29 and 30, and lines 29 and 36 are cut. To observe how the accuracy of our approximation changes with blackout severity, we increased the generation at the generation-rich region in the lower subsystem and load in the load rich region. For each of these test cases, the excess generation and load were equally distributed among the generators and load nodes in the respective regions. The results of our experiments are presented in Fig. 6.2.

In Fig. 6.2, the horizontal axis correspond to the estimated size of a blackout, which we compute by (4.12). The vertical axis correspond to the measured size of a blackout, which we compute by solving the optimal load shedding problem in (3.18)(3.33). To solve the associated nonlinear optimization problem, we used Matlab's 


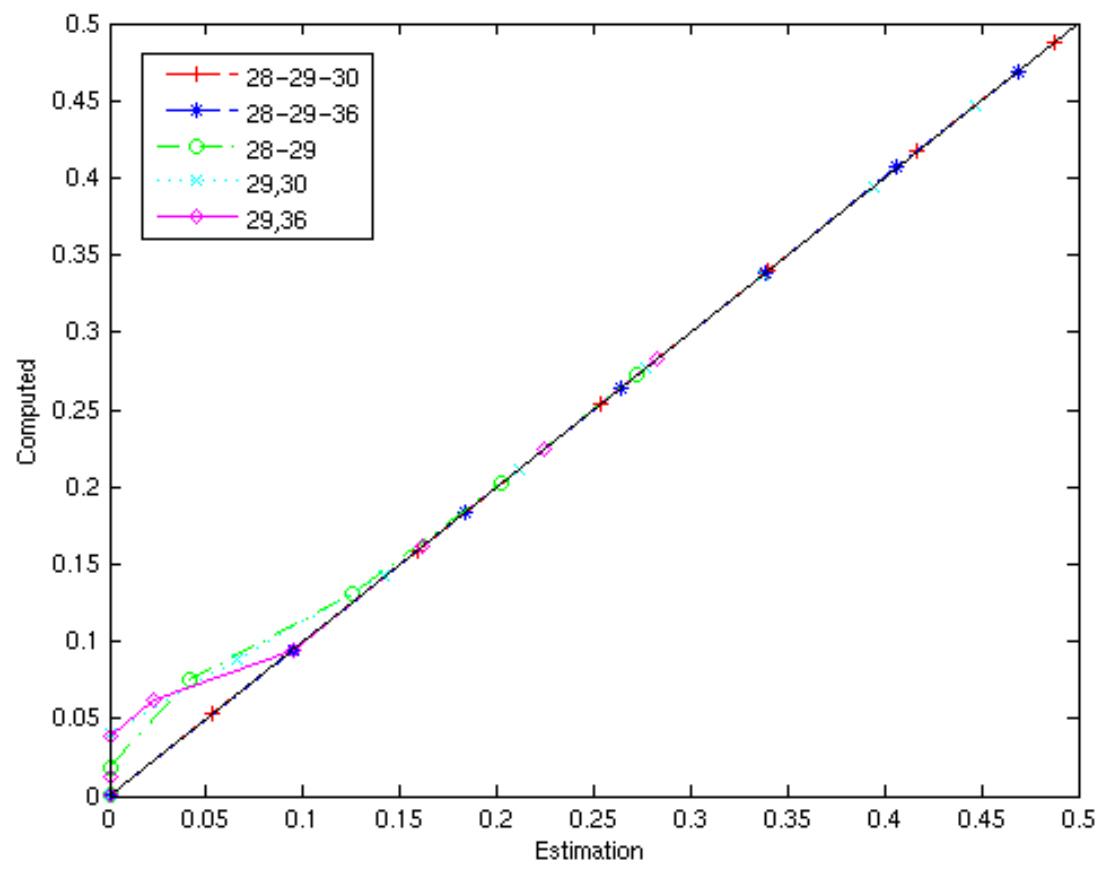

FIG. 6.2. Accuracy of our combinatorial approximation to the MINLP formulation.

fmincon with default settings. Both axes are normalized with respect to the total generation in the system. Each data point corresponds to an experiment with a specified set of broken lines, and a generation/load assignment. Experiments with the same set of broken lines are marked with the same sign. Ideally, all points should lie on the main diagonal, which means the estimation is equal to the measurement. However, this does not always hold, either due to the imperfectness of our approximation, or due to the imperfectness of the nonlinear solver we use to solve the load shedding problem. Note that both factors cause the measured value to be higher than the estimated value, since our approximation is always an underestimation, and the solver is bound to find local optima for our nonconvex optimization problem.

The results show that our approximation works effectively, especially for blackouts with high severity. The first two sets of broken lines, leave only a single line between the generation-rich and load-rich regions. By our analysis, we know that this line will be saturated, and thus our combinatorial approximation will be exact. We can see that the empirical results are consistent with our theoretical studies. The other three cases leave two lines between the two regions. The results show that our combinatorial model underestimates the severity of a blackout (sometimes even misses a blackout, which correspond to the points on the vertical axis), but the gap between estimated and measured values closes rapidly as the blackout severity increases. Note that we are only interested in severe blackouts, and thus our combinatorial approximation is accurate for our purposes.

In the second set of experiments, we applied our integer programming formulation for the network inhibition problem to identify vulnerabilities of a simplified model of 
the Western states power grid with 13,374 nodes and 16,520 lines. We used PICO, a massively parallel integer programming solver, developed by Phillips et al. at Sandia National Laboratories, to solve the associated MILPproblems. The results of our experiments are presented in Fig.6.3. While PICO can be run in parallel, we performed our experiments on a single processor. As seen in this figure severity increases with

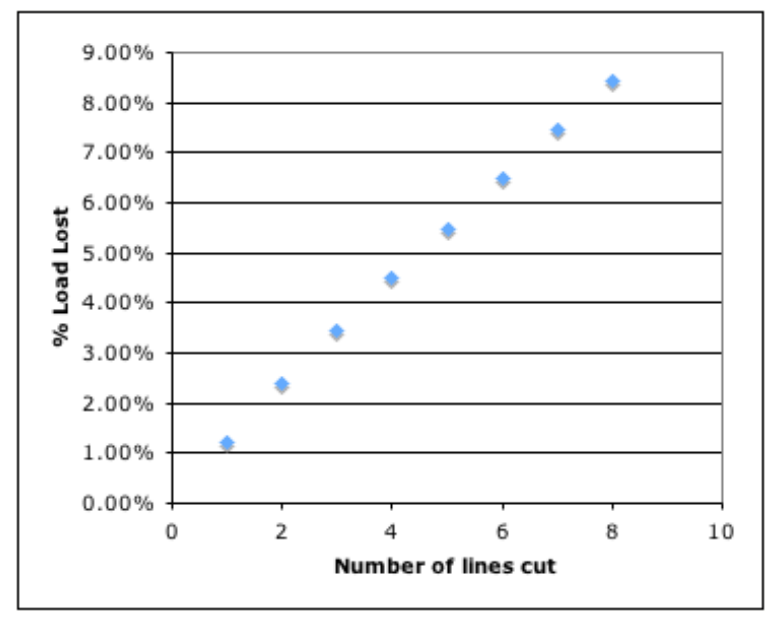

FIG. 6.3. Results on a grid with 13,374 nodes and 16,520 edges, which is a simplified model for the Western United States power grid

number of lines being cut. The solution times for these problems were only in the order of tens of seconds on an Opteron $2.2 \mathrm{GHz}$ processor with 4.4 GFlops/sec theoretical peak and 6 Gbytes of physical memory. The main conclusion, we can draw from these experiments is that our reduction from the MINLP formulation of the power grid vulnerability analysis problem to the MILP formulation of the network inhibition problem grants practical solutions. The associated integer programming formulations for the network inhibition problem can be applied for the vulnerability analysis of real-world problems.

7. Conclusions and future work. We studied the problem of identifying small groups of lines, whose removal would cause a severe blackout, which is critical for the secure operation of the electric power grid. We first presented a mixed integer nonlinear programming (MINLP) formulation of the problem. Our analysis of this formulation revealed a special combinatorial structure that we exploited to avoid nonlinearity and approximate the original problem as a pure combinatorial problem. The key new observation behind our analysis was the correspondence between the Jacobian matrix (a representation of the feasibility boundary of the equations that describe the flow of power in the network) and the Laplacian matrix in spectral graph theory (a representation of the graph of the power grid). Our reduction allowed us to directly seek values of discrete variables in an optimal solution to our MINLP formulation, without explicitly solving the nonlinear part, which simplifies the problem complexity theoretically and practically. The reduced combinatorial problem is known as the network inhibition problem, for which we presented a mixed integer linear programming formulation. Our empirical studies showed that our combinatorial approximation is accurate, especially as the blackout size increases, and we can solve the corresponding integer programming problems even for tens of thousands of lines. 
This work leads to new research problems both for optimization and for power systems communities. First, we have used a simplified model of power flow equations, where the fixed the voltages and focused only at active power. Our formulation can be easily extended to include reactive power, but our analysis of an optimal solution needs to be revised. It remains to be seen if there exists a combinatorial structure when the reactive power is also included in the analysis. Another interesting question is whether it is possible to find tighter bounds for the flow between two regions. We believe our current bound of cumulative sum of capacities of active lines can be improved by careful analysis of power flow equations, which will be an interesting question, especially for power systems experts. Finally, it would be interesting to include system dynamics in vulnerability analysis, without solving differential algebraic equations. Stochastic models might be useful in this context.

On the optimization front, solving the MINLP formulation remains as a challenge. Especially, using our discrete approximation within a decomposition method needs to be investigated. Recall that our approximation is more than merely solving for binary variables, where continuous variables for the nonlinear part are fixed. The reduced problem can foresee changes in the nonlinear part, and directly seek values of binary variables (i.e., the broken lines in this problem) in an optimal solution. Our reduction can still be used to accelerate a decomposition algorithm. We expect that such a study to be closely related to how flow between two regions can be bounded. Improved solution methods for the network inhibition problem, especially when additional constraints that better bound the flow between two regions, is another interesting problem to study. Both heuristics and exact algorithms would be interesting in this context.

\section{REFERENCES}

[1] Power system test case archive. available at http://www.ee.washington.edu/research/pstca/.

[2] F. Alvarado, I. Dobson, And Y. Hu, Computation of closest bifurcations in power systems, IEEE Transactions on Power Systems, 9 (1994), pp. 918-928.

[3] D. Bienstock and S. MatTia, Using mixed-integer programming to solve power grid blackout problems, Tech. Rep. CORC Report TR-2005-07, Dept. of IEOR, Columbia University, 2005.

[4] B. Carreras, V. Lynch, I. Dobson, and D. Newman, Dynamics, criticality, and selforganization in a model for blackouts in power transmission systems, in Proc. 35th Hawaii International Conference on System Sciences, Hawaii, 2002.

[5] - Dynamical and probabilistic approaches to the study of blackout vulnerability of the power transmission grid, in Proc. 37th Hawaii International Conference on System Sciences, Hawaii, 2004.

[6] B. Carreras, V. Lynch, M. Sactuen, I. Dobson, and D. Newman, Modeling blackout dynamics in power transmission networks with simple structure, in Proc. 34th Hawaii International Conference on System Sciences, Maui, Hawaii, 2001.

[7] T. Cormen, C. E. Leiserson, R. L. Rivest, and C. Stein, Introduction to Algorithms, The MIT Press, 2001.

[8] I. Dobson, B. Carreras, V. Lynch, and D. Newman, An initial model for complex dynamics in electric power system blackouts, in Proc. 34th Hawaii International Conference on System Sciences, Maui, Hawaii, 2001.

[9] I. Dobson, J. Chen, J. Thorp, B. Carreras, and D. Newman, Examining criticality of blackouts in power system models with cascading events, in Proc. 35th Hawaii International Conference on System Sciences, Hawaii, 2002.

[10] I. Dobson, K. Wierzbicki, B. Carreras, V. Lynch, and D. Newman, An estimator of propagation of cascading failure, in Proc. 39th Hawaii International Conference on System Sciences, Hawaii, 2006.

[11] V. Donde, V. Lopez, B. Lesieutre, A. Pinar, C. Yang, and J. Meza, Identification of severe multiple contingencies in electric power systems, IEEE Transactions on Power Systems. to 
appear.

[12] - Identification of severe multiple contingencies in electric power networks, in Proceedings of the 37th North American Power Symposium, Ames, Iowa, 2005.

[13] M. Fiedler, Algebraic connectivity of graphs, Czech. Math. Journal, 23 (1973), pp. 298-305.

[14] A. V. Goldberg and S. RaO, Beyond the flow decomposition barrier, Journal of ACM, 45 (1998), pp. 783-797.

[15] S. Grijalva And P. Sauer, Static collapse and topological cuts, in Proc. 38th Hawaii International Conference on System Sciences, Hawaii, 2005.

[16] S. GrJialva And P. Sauer, A necessary condition of power jacobian singularity based on branch complex flows, IEEE Transactions on Circuits and Systems-I: Regular Papers, 52 (2005), pp. 1406-1413.

[17] T. He, S. Kolluri, S. Mandal, F. Galvan, and P. Rastgoufard, Identification of weak locations in bulk transmission systems using voltage stability margin index, in Applied Mathematics for Restructured Power Systems: Optimization, Control, and Computational Intelligence, New York, 2005, Springer.

[18] J.Arroyo and F. Galiana, On the solution of the bilevel programming formulation to the terrorist threat problem, IEEE Transactions on Power Systems, 20 (2005), pp. 789-797.

[19] B. Lesieutre, A. Pinar, And S. Roy, Power system extreme event detection: The vulnerability frontier, in Proc. 41st Hawaii International Conference on System Sciences, Hawaii, 2008. to appear.

[20] B. Lesieutre, S. Roy, V. Donde, And A. Pinar, Power sytem extreme event screening using graph partitioning, in Proceedings of the 38th North American Power Symposium, Carbondale, Illinois, 2006.

[21] G. Oliviera, S. Binato, L. Bahiense, L. Thome, and M. Pereira, Security-constrained tranmission planning: a mixed-integer disjunctive approach, in Optimization Online, 2004.

[22] C. A. Phillips, The network inhibition problem, in STOC '93: Proceedings of the twenty-fifth annual ACM symposium on Theory of computing, New York, NY, USA, 1993, ACM Press, pp. $776-785$.

[23] A. Pinar, Y. Fogel, And B. Lesieutre, The inhibiting bisection problem, Tech. Rep. Technical Report LBNL-62142, Lawrence Berkeley National Laboratory, 2006.

[24] J. ROYSET AND R. WOOD, Solving the bi-objective maximum-flow network-interdiction problem, INFORMS Journal on Computing, 19 (2007), pp. 175-184.

[25] J. Salmeron, K. Wood, and R. Baldick, Analysis of electric grid security under terrorist threat, IEEE Transactions on Power Systems, 19 (2004), pp. 905-912.

[26] R. E. TARJAn, Data Structures and Network Algorithms, Siam Press, 1998. 